\title{
TREM-1-expressing intestinal macrophages crucially amplify chronic inflammation in experimental colitis and inflammatory bowel diseases
}

\author{
Mirjam Schenk, ${ }^{1}$ Axel Bouchon, ${ }^{2}$ Frank Seibold, ${ }^{3}$ and Christoph Mueller ${ }^{1}$ \\ 1Division of Immunopathology, Institute of Pathology, University of Bern, Bern, Switzerland. 2Bayer HealthCare, Wuppertal, Germany. \\ ${ }^{3}$ Division of Gastroenterology, Inselspital, University of Bern, Bern, Switzerland.
}

\begin{abstract}
Triggering receptor expressed on myeloid cells-1 (TREM-1) potently amplifies acute inflammatory responses by enhancing degranulation and secretion of proinflammatory mediators. Here we demonstrate that TREM-1 is also crucially involved in chronic inflammatory bowel diseases (IBD). Myeloid cells of the normal intestine generally lack TREM-1 expression. In experimental mouse models of colitis and in patients with IBD, however, TREM-1 expression in the intestine was upregulated and correlated with disease activity. TREM-1 significantly enhanced the secretion of relevant proinflammatory mediators in intestinal macrophages from IBD patients. Blocking TREM-1 by the administration of an antagonistic peptide substantially attenuated clinical course and histopathological alterations in experimental mouse models of colitis. This effect was also seen when the antagonistic peptide was administered only after the first appearance of clinical signs of colitis. Hence, TREM-1-mediated amplification of inflammation contributes not only to the exacerbation of acute inflammatory disorders but also to the perpetuation of chronic inflammatory disorders. Furthermore, interfering with TREM-1 engagement leads to the simultaneous reduction of production and secretion of a variety of pro-inflammatory mediators such as TNF, IL-6, IL-8 (CXCL8), MCP-1 (CCL2), and IL-1 $\beta$. Therefore, TREM-1 may also represent an attractive target for the treatment of chronic inflammatory disorders.
\end{abstract}

\section{Introduction}

Triggering receptor expressed on myeloid cells-1 (TREM-1) was identified by screening for transmembrane receptors on myeloid cells associated with the activating ITAM-containing adaptor molecule DAP12 (1). TREM-1 belongs to the immunoglobulin superfamily and is part of a gene cluster encoding several TREM and TREM-like molecules that share structural elements but have low amino acid homology (1). TREM-1 is constitutively expressed on most monocytes/macrophages and neutrophils and is upregulated by various stimuli such as the TLR ligands LPS and lipoteichoic acid (LTA) and the proinflammatory cytokine TNF $(2,3)$. Hence, it has been suggested that TREM-1 is mainly involved in acute inflammatory reactions but not in chronic inflammatory disorders (2). The natural ligand of TREM-1 is still unknown. Agonistic antiTREM-1 antibodies have been used to determine the functional consequences of receptor engagement and the signaling pathways (4). Activation of TREM-1 on monocytes and neutrophils enhances secretion of inflammatory cytokines and chemokines and leads to upregulation of cell-surface molecules involved in extravasation, cell activation, and costimulation (4). Proinflammatory cytokine production and lethality in mouse models of septic shock are reduced by blocking TREM-1 with a recombinant TREM-1 fusion protein (mTREM-1-hFcy1) (2) or a synthetic antagonistic peptide

Nonstandard abbreviations used: CD, Crohn disease; DSS, dextran sodium sulfate; IBD, inflammatory bowel disease; sTREM-1, soluble form of TREM-1; TREM-1, triggering receptor expressed on myeloid cells-1; UC, ulcerative colitis.

Conflict of interest: The authors have declared that no conflict of interest exists. Citation for this article: J. Clin. Invest. 117:3097-3106 (2007). doi:10.1172/JCI30602.
(5). Moreover, bacterial sepsis induces the release of a soluble form of TREM-1 (sTREM-1) in human patients and mouse models. Serum levels of sTREM-1 positively correlate with survival $(6,7)$, thus indicating an antagonistic effect of sTREM-1.

Monocytes and macrophages obtained from secondary lymphoid organs mostly express functional TREM-1 on the cell surface. In contrast, the vast majority of the resident intestinal macrophages completely lack expression of TREM-1, possibly as a further adaptation to the antigen-rich microenvironment of the intestinal mucosa. This differentiation status of the intestinal macrophages may be maintained by the local presence of the immunomodulatory cytokines IL-10 and TGF- $\beta$, which synergistically downregulate TREM-1 (3). Since an aberrant immune reaction against luminal antigens including commensal bacteria is generally considered a main disease-promoting factor in the pathogenesis of inflammatory bowel diseases (IBD) (8-10), we hypothesized that in patients with active Crohn disease (CD) and ulcerative colitis (UC), TREM-1 expression would be upregulated on intestinal macrophages and that aberrant expression of TREM-1 in the intestinal mucosa may contribute to the excessive inflammatory reactions and tissue destruction typically seen in the affected intestinal mucosa of patients with active IBD.

\section{Results}

TREM-1-expressing macrophages are significantly increased in the inflamed mucosa of IBD patients. As previously shown (3), lamina propria macrophages from the normal small and large intestine generally do not express TREM-1, and the few TREM-1-positive cells that are present may represent recently recruited blood monocytes (Figure 1A). 
A
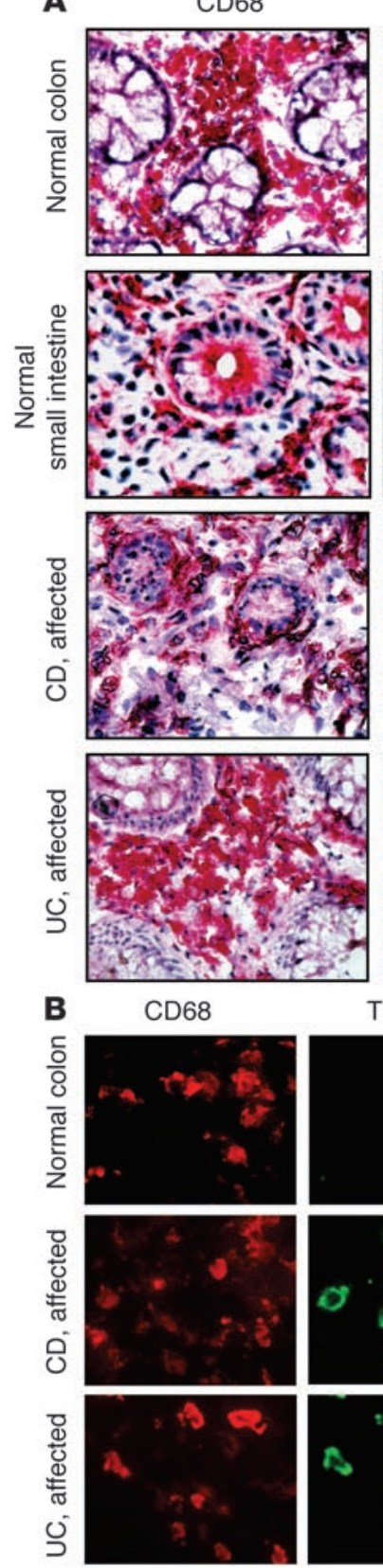

TREM-1
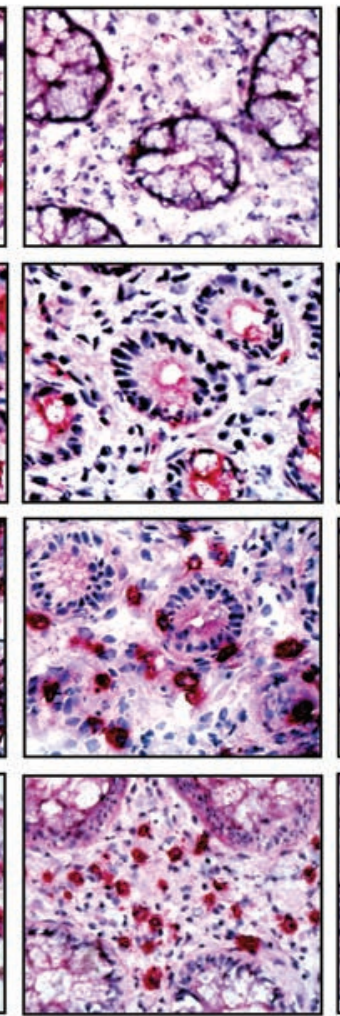

TREM-1
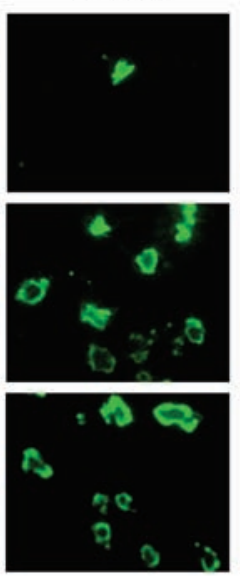
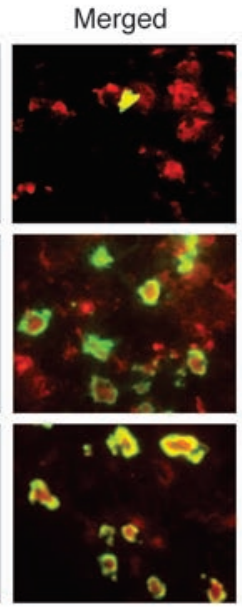

Isotype ctrl
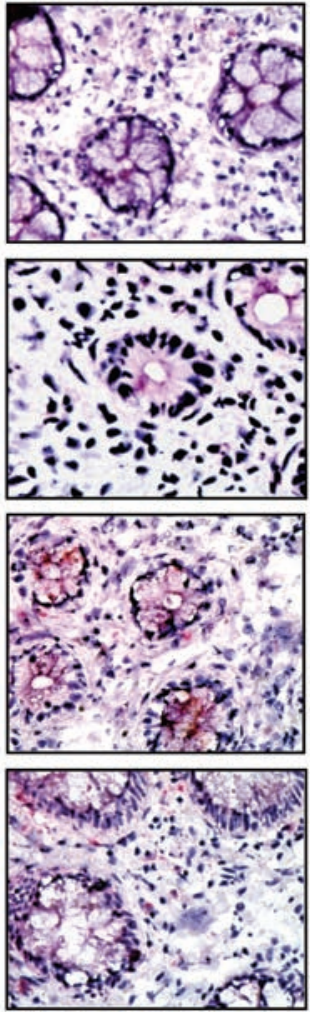

Isotype ctrl
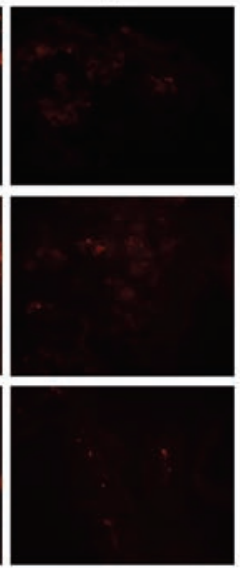

Figure 1

TREM-1-expressing macrophages are increased in the intestinal lamina propria of IBD patients. (A) Serial frozen sections of the intestine from normal donors and IBD (CD and UC) patients with active disease were immunostained for TREM-1 and CD68. An isotype-matched irrelevant $\mathrm{mAb}$ was used as a negative control (Isotype ctrl). Stainings are representative of at least 12 different intestinal tissue samples for the normal small and large intestine and of at least 10 tissues each for UC and CD. Original magnification, $\times 40$. (B) Double immunofluorescence stainings demonstrate that most TREM-1positive cells (green) in the intestinal lamina propria from patients with active IBD (UC and $\mathrm{CD}$ ) were $\mathrm{CD}^{+} 8^{+}$macrophages (red). Original magnification, $\times 100$.

The absence of TREM-1 expression on lamina propria macrophages in the normal intestine is likely to prevent overwhelming inflammatory reactions and thus excessive tissue damage (11). To assess whether aberrant TREM-1 expression on myeloid cells

may contribute to inflammatory reactions in the intestine, we screened intestinal tissue samples from patients with inflammatory conditions for TREM-1-expressing macrophages. The number of TREM-1-positive macrophages was increased in the inflamed intestine of patients with acute appendicitis and in patients with acute diverticulitis (Supplemental Figure 1; supplemental material available online with this article; doi:10.1172/ JCI30602DS1). While these findings were expected due to the acute inflammatory process, we also detected increased frequencies of TREM-1-expressing cells on immunostained sections from patients with chronic IBD (Figure 1A). Double immunofluorescent stains of frozen tissue sections using CD68 as a cytoplasmic marker for macrophages and the membrane-associated TREM-1 showed that the majority of TREM-1-expressing cells in the affected intestine of IBD patients are intestinal macrophages (Figure 1B); while occasionally TREM-1-expressing neutrophils were also found (Supplemental Figure 2), they invariably represented less than $20 \%$ of the total TREM-1-positive cells (data not shown).

To characterize and quantify the TREM-1-positive macrophages present in the inflamed intestinal mucosa of IBD patients, we determined the expression profile of additional cell-surface receptors upon their ex vivo isolation. Whereas only a few TREM-1positive macrophages were present in the normal intestinal mucosa, this macrophage subset was substantially more abundant in the affected lesions of patients with IBD, and usually $30 \%-40 \%$ of the intestinal macrophage population isolated from both UC and CD patients expressed cell-surface TREM-1 (Figure 2A). As shown in Figure 2B, TREM-1-positive macrophages also coexpressed CD14 and CD89, i.e., 2 cell surface receptors that are generally absent on macrophages from normal intestinal tissue samples. No difference in the frequency or expression level of TREM-1 on intestinal macrophages from the affected mucosa of patients with UC and $\mathrm{CD}$ was found. The combined results for 18 patients with active IBD revealed an approximately 5 -fold increase in the frequency of TREM-1-expressing macrophages in the affected lesions when compared with normal control tissues (Figure 2C).

In IBD patients, aberrant TREM-1 expression on intestinal macrophages mediates enhanced secretion of proinflammatory cytokines and chemokines. To determine the possible consequences of TREM-1 expression directly, intestinal macrophages were isolated from tissue samples and cultured in vitro for 24 hours in the presence or absence of an agonistic TREM-1binding $\mathrm{mAb}$. As shown in Figure 3, engagement of TREM-1 resulted in increased secretion of the proinflammatory mediators TNF, monocyte chemoattractant protein-1 (MCP-1) (CCL2), IL-6, IL-8 (CXCL8), and IL-1 $\beta$. Although the secretion of these proinflammatory cytokines and chemokines was already elevated in macrophages from inflamed intestinal 
A Normal colon
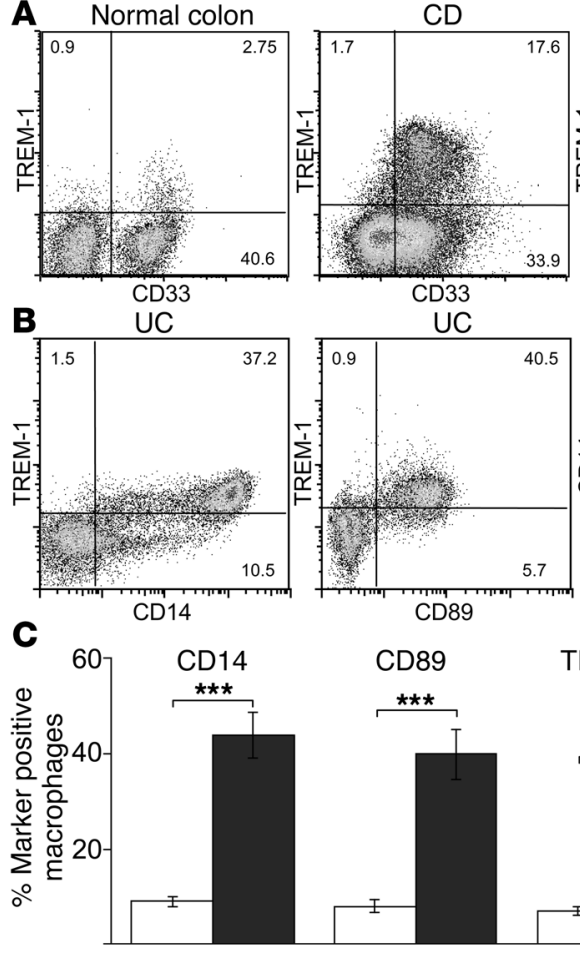

UC
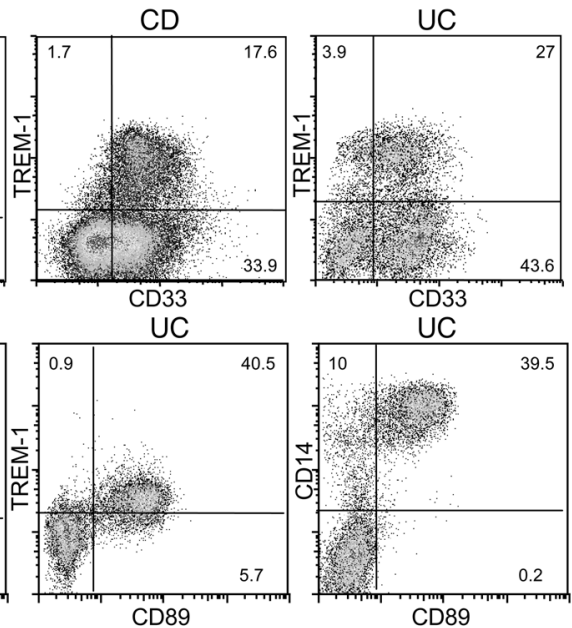

UC
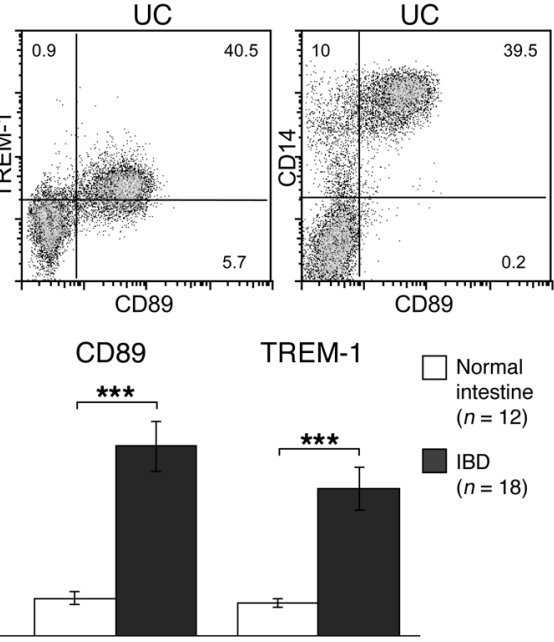

mucosa of IBD compared with normal tissues, we still observed significantly increased secretion after TREM-1 crosslinking from inflamed but not normal control tissue specimens (Figure 3). Hence, TREM-1-mediated amplification of the inflammatory process likely contributes to the exacerbation of disease and tissue destruction in patients with IBD.

Extent of TREM-1 expression in the intestinal mucosa is related to disease activity in clinical and experimental IBD. Since such a dramatic increase in the frequency of TREM-1-expressing macrophages was seen in the affected intestinal areas of patients with IBD, we determined how expression of TREM- 1 is related to disease activity in patients

\section{Figure 2}

TREM-1-expressing macrophages are increased in the affected intestinal mucosa of patients with IBD and coexpress CD14 and CD89. (A) Lamina propria macrophages of normal intestinal tissue specimens and of patients with active IBD were isolated and analyzed by FACS for TREM-1, CD14, and CD89 cell-surface expression. (B) Coexpression of CD14 and CD89 on TREM-1-positive intestinal macrophages is shown for a representative UC patient. (C) The frequency of TREM-1-expressing lamina propria macrophages is significantly increased in patients with IBD ( $n=18$ : UC, $n=7$; CD, $n=11)$ compared with normal individuals $(n=12)$. Percentages are indicated as mean \pm SEM; ${ }^{\star \star \star} P<0.001$.

with IBD. To this end, we compared TREM-1 mRNA expression levels in intestinal mucosal samples from affected and unaffected sites in patients with active CD and UC, samples from patients with inactive disease, and normal control tissue specimens. In the normal human intestine, TREM-1 mRNA was generally absent and only occasionally detectable by real-time PCR, whereas it was consistently expressed at high levels in affected segments from $C D$ and UC patients with active disease (Figure 4, A and B). In the macroscopically unaffected tissue samples obtained from sites adjacent to the affected area in patients with $\mathrm{CD}$ and in tissue samples with inactive disease from patients with UC, TREM-1 mRNA expression was substantially reduced and barely detectable, whereas TNF mRNA was detected at comparable levels in active and inactive disease samples from the same patients (Figure 4A).

The close association of TREM-1 mRNA expression with signs of disease activity in IBD prompted us to follow TREM-1 during the early stages of experimental colitis. Therefore, we analyzed TREM-1 induction in the CD4 $\mathrm{T}^{+}$cell transfer model of colitis in lymphopenic $\mathrm{RAG}^{-/-}$recipients $(12-14)$ and the $\mathrm{T}$ cell-independent dextran sodium sulfate (DSS) model in C57BL/6 mice (15-18). Colonic tissue samples, collected on different days upon colitis induction, were analyzed for TREM-1 and TNF expression at mRNA and protein levels, using RT-PCR and Western blot analysis, respectively (Figure 5, A and B). TREM-1 was generally not detectable on mRNA and protein levels in the normal colon of C57BL/ 6 and RAG2 ${ }^{-/-}$mice (Figure 5, A and B, day 0). However, TREM-1 was

\section{Figure 3}

Secretion of TNF, IL-6, IL-8 (CXCL8), monocyte chemoattractant protein-1 (MCP-1 [CCL2]), and IL-1 $\beta$ is significantly enhanced following engagement of TREM-1 on intestinal lamina propria macrophages from IBD but not from normal control patients. Intestinal macrophages isolated from CD patients $(n=11)$, UC patients $(n=7)$, and normal control patients $(n=5)$ were cultured for 24 hours in the presence (black bars) or absence (white bars) of an agonistic plate-bound anti-TREM-1 mAb. Cytokine concentrations were measured simultaneously in the supernatant. Data represent mean $\pm \mathrm{SEM}$; ${ }^{*} P<0.05$.
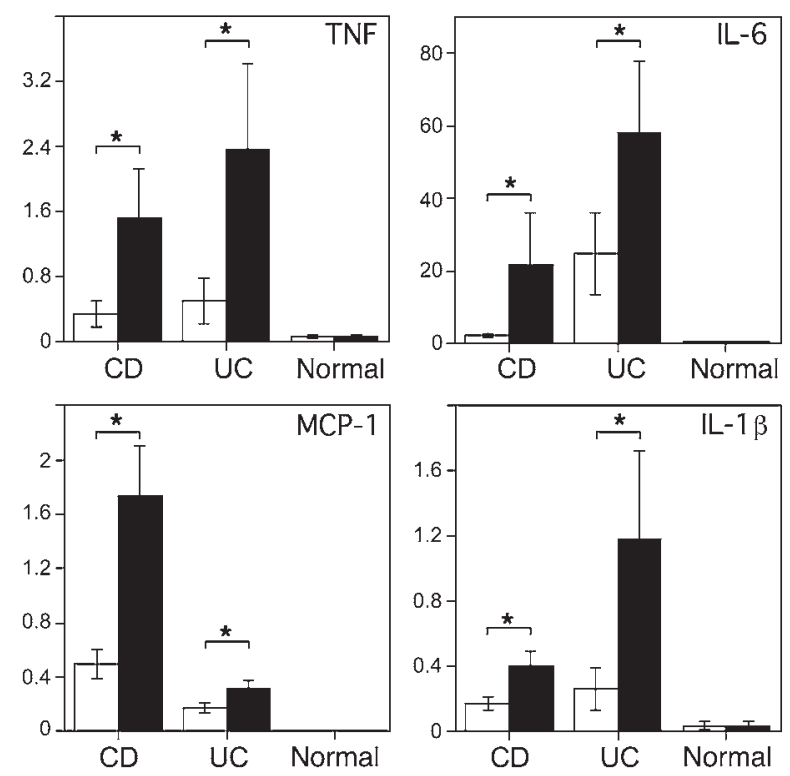

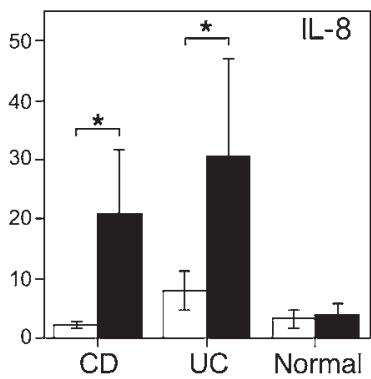

Cytokines (ng/ml) detected in the supernatant $24 \mathrm{~h}$ after TREM-1 crosslinking

Cytokines (ng/ml) detected in the supernatant $24 \mathrm{~h}$ after addition of isotype-matched $\mathrm{mAb}$ 
A
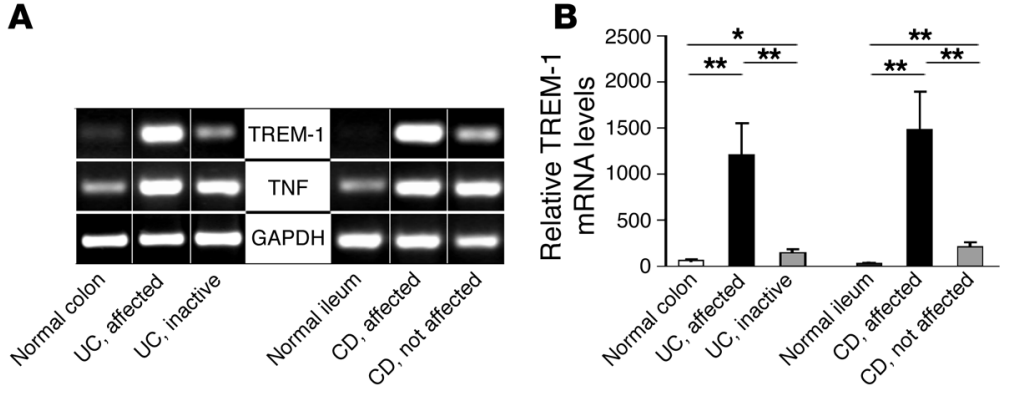

Figure 4

Enhanced disease activity is associated with increased TREM-1 mRNA expression in the intestinal mucosa of patients with IBD. RNA was isolated from endoscopic biopsies of actively inflamed intestinal mucosa and of macroscopically nonaffected areas from CD and UC patients and from normal control samples. (A) Semiquantitative RT-PCR was performed for TREM-1 and TNF. Amplification of GAPDH is shown as an internal control. One representative example is shown for 5 normal, $11 \mathrm{CD}$, and 7 UC patients analyzed. (B) TREM-1 mRNA levels were analyzed by quantitative RT-PCR. The cycle number at which the TREM-1 transcripts were detectable was compared with that of GAPDH as an internal control and expressed as arbitrary units; indicated are the mean values ( \pm SEM) from at least 6 tissue samples per group. ${ }^{* \star} P<0.01 ;{ }^{*} P<0.05$.

upregulated as a very early event during colitis induction, preceding the first histopathological signs of colitis seen after 5 days in the transfer model and after 3 days in the DSS model (Figure 5, A and C). Intriguingly, the increase in TREM-1 mRNA expression level was closely related to enhanced disease activity in both mouse models of colitis. TREM-1 mRNA expression also correlated well with the detection of TREM-1 protein, as demonstrated in the Western blot prepared from colonic tissue samples (Figure 5B). Furthermore, immunostaining for TREM-1 and CD11b (Mac-1) confirmed the correlation between TREM-1-expressing macrophage frequency and severity of histopathological signs of colitis (Supplemental Figure 3). Hence, TREM-1 upregulation is an early event in the pathogenesis of experimental colitis and enhanced disease activity is associated with increased TREM-1 expression during experimental and clinical IBD.

Blocking TREM-1 attenuates intestinal inflammation in animal models of colitis. The early upregulation of TREM-1 in the affected tissue during experimental induction of colitis prompted us to assess how interfering with TREM-1 activation affects disease severity in experimental colitis. To prevent TREM-1 engagement, we used an antagonistic synthetic peptide, derived from the extracellular domain of TREM-1. This peptide (LP17) was originally designed by Gibot et al. (5) and protects mice from LPS-induced lethality. The LP17 peptide used in our experiments significantly attenuated the increase in TNF serum levels following the administration of LPS in C57BL/6 mice (Supplemental Figure 4), verifying that the LP17 peptide was affecting TREM-1 activation in vivo. Upon induction of colitis by either the administration of DSS into the drinking water of C57BL/ 6 mice or the transfer of colitogenic $\mathrm{CD}^{+} \mathrm{T}$ cells into $\mathrm{RAG} 2^{-/-}$recipients, mice were treated either with an antagonistic TREM-1 peptide (LP17) or with a sequence-scrambled control peptide. In experimental animals the extent of diarrhea was followed using a semiquantitative scoring system including detection of occult blood in the feces and rectal bleeding (see Methods). At the end of the observation period, mice were sacrificed, and part of the colon was paraffin embedded for subsequent detailed histopathological analyses. In the LP17-treated group, the number of mice with occult blood in the feces and

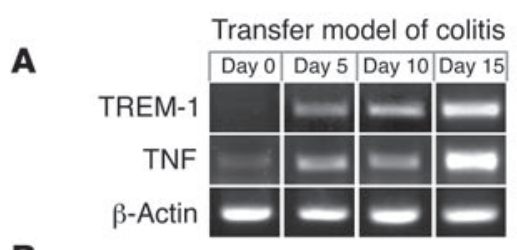

B

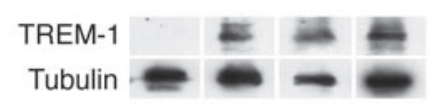

C

Day 0
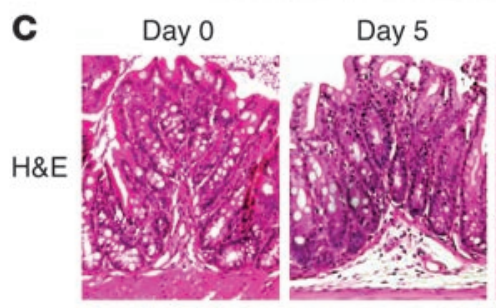

Day 15

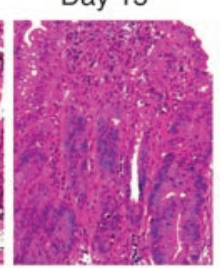

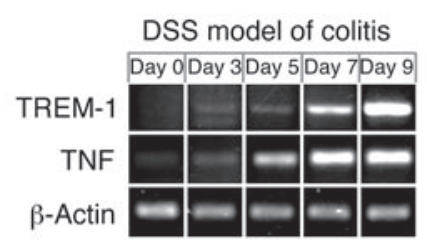
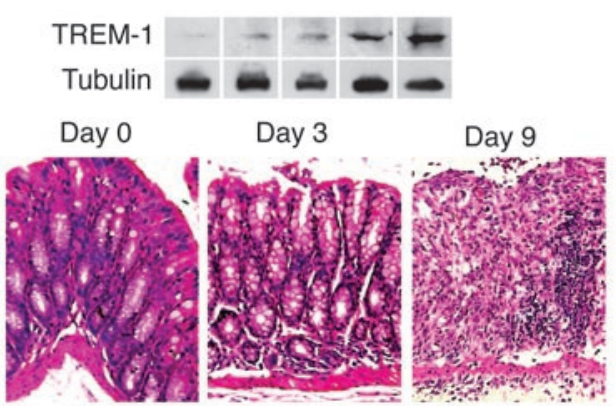

\section{Figure 5}

TREM-1 mRNA and protein expression in the colon correlates with colonic inflammation and is induced early during onset of experimental colitis. (A) Kinetics of TREM-1 and TNF transcription in the colon upon colitis induction by CD4+ $\mathrm{T}$ cell transfer into RAG2 ${ }^{-/-}$recipient mice (transfer model) and the DSS mouse model of intestinal inflammation in C57BL/6 mice. $\beta$-Actin was used as a loading control. (B) Western blot analyses of colonic tissue samples adjacent to those used for RNA isolation demonstrate the increased TREM-1 protein expression during induction of colitis. Tubulin is shown as a control. (C) H\&E-stained paraffin-embedded tissue sections demonstrate the onset of histopathological alterations after 5 days after cell transfer and after 3 days after DSS administration. Original magnification, $\times 40$. In each model 4 mice were analyzed per time point in 2 independent experiments. 
A Extent of diarrhea

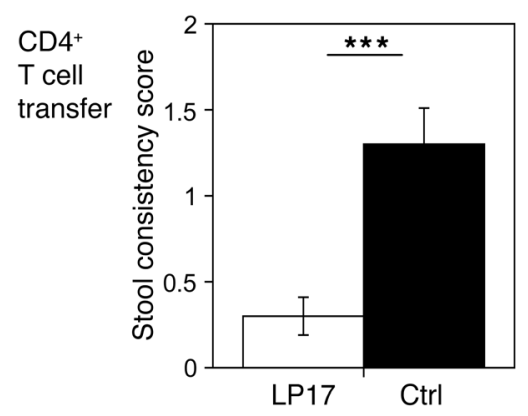

DSS

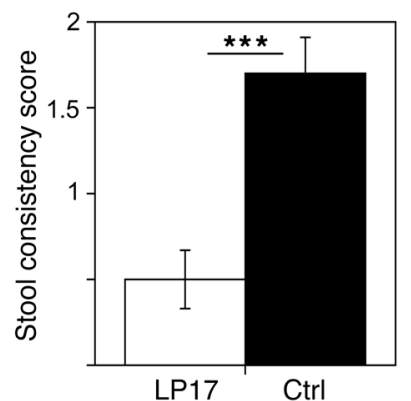

B Shortening of the colon
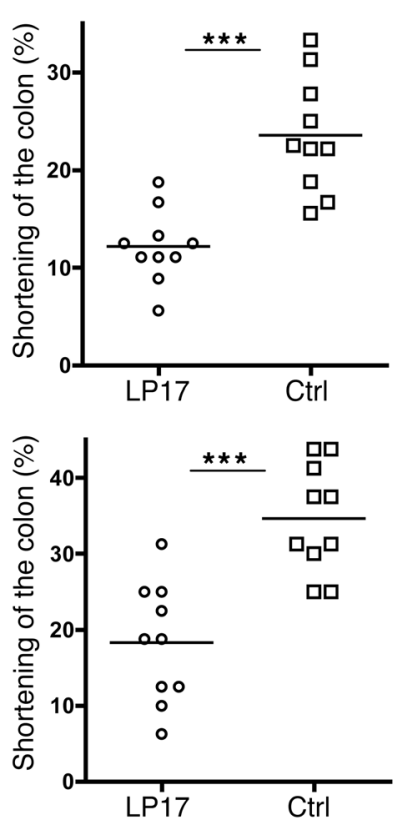

C Histopathology score
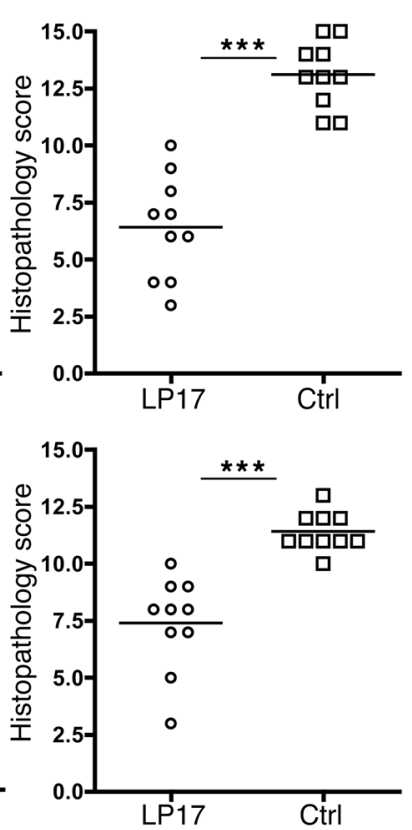

D

RT-PCR analysis
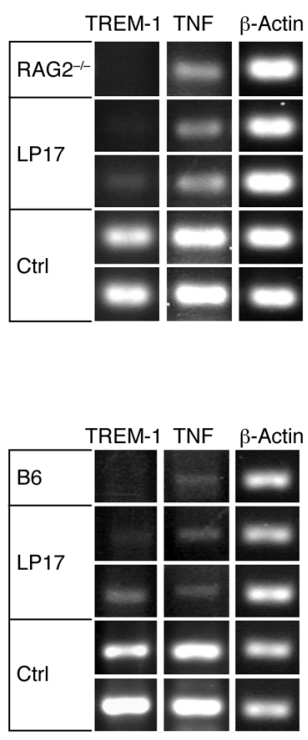

\section{Figure 6}

Blocking TREM-1 attenuates intestinal inflammation in 2 distinct mouse models of colitis. Colitis was induced either by the transfer of splenic $\mathrm{CD}^{+}{ }^{+} \mathrm{CD} 45 \mathrm{RB}^{\mathrm{hi}} \mathrm{T}$ cells into RAG2 ${ }^{-/-}$recipients (top row) or by oral administration of DSS (bottom row). Upon colitis induction, experimental mice were treated daily either with an antagonistic TREM-1 peptide (LP17) or with a control peptide. (A) Fecal samples from each mouse were tested for the presence of occult blood and bloody diarrhea to obtain the stool consistency score (see Methods). Data are presented as mean \pm SEM; $n=10$. (B) The colon length of each mouse was measured from the end of the cecum to the anus; indicated are the mean loss of colon length (\%) at the end of the experiment. (C) The colitis scores for the 2 groups (LP17-treated and control-treated) were determined as described in Methods. (D) PCR analysis for TREM-1 and TNF mRNA was performed on identical segments of the distal colon in untreated mice (RAG2 ${ }^{-1-}$; C57BL/6 [B6]) and antagonistic TREM-1- (LP17-) and control peptide-treated mice upon colitis induction. $\beta$-Actin was used as loading control. Results for 1 representative unmanipulated control mouse and 2 representative experimental mice per group are shown. ${ }^{* * *} P<0.001 ; n=10$.

rectal bleeding was significantly reduced compared with the control-treated animals (Figure 6A). Generally, in both mouse models of colitis, the LP17 treatment significantly attenuated the shortening of the colon by approximately 50\% (Figure 6B).

To quantitate the histological alterations present in the colon of experimental mice, a semiquantitative colitis scoring system ranging from 0 (no histopathological alterations) to 15 (most severe histopathological alterations) was established (see Methods). The results of these histopathological analyses of colonic tissue sections are summarized in Figure 6C. Control-treated animals in both models revealed severe histopathological alterations of the colon; LP17-treated mice, however, showed only moderate signs of histopathological alterations, with a significantly lower colitis score (Figure 6C).

Consistent with the data in patients with active IBD, TNF and TREM-1 mRNA expression were increased in the affected colon of colitic mice and correlated with disease activity (Figures 4 and 5). Hence, we also compared TREM-1 and TNF mRNA expression levels in the LP17-treated and control-treated colitic mice. Tissue samples from identical distal parts of the colon from all mice were used for analysis. TREM-1 mRNA expression was generally absent in the colon of normal RAG2 ${ }^{-/-}$and C57BL/6 mice, whereas low TNF mRNA expression was detected (Figure 6D). Colitic mice treated with the control peptide invariably showed strong induction of TREM-1 and TNF mRNA in the affected colon. LP17-treat- ed mice, however, showed low levels of TNF mRNA and TREM-1 mRNA (Figure 6D), confirming the inflammation-attenuating effects of blocking TREM-1 engagement in vivo.

TREM-1 may represent an attractive target for the treatment of chronic inflammatory disorders. Finally, we wanted to assess whether TREM-1 inhibition can also correct intestinal inflammation after onset of colitis. To perform these experiments, we used the DSS model of colitis, as it shows highly reproducible kinetics of disease induction. On day 3 after DSS administration, the mice had persistent diarrhea and bloody stools (detected by hemo FEC) and showed early histopathological signs of colitis. Based on these signs, we analyzed a baseline group of animals on day 3 and started treatment with either an antagonistic TREM-1 peptide (LP17) or a sequencescrambled peptide (control) until analysis on day 9 (Figure 7A). In the LP17-treated group, the number of mice with occult blood in the feces and rectal bleeding was significantly reduced compared with the control-treated animals and the mice analyzed on day 3 (Figure 7B). The LP17-treated mice also showed less colonic shortening compared with the control-treated mice (Figure 7C).

The histopathological scores obtained on day 3 of DSS administration confirmed the onset of an inflammatory reaction in the colonic mucosa at this time. At the end of the observation period, experimental mice that received DSS but in which TREM-1-TREM-1L interactions were left intact revealed massive alterations of the colon. In contrast, experimental mice that were 
A

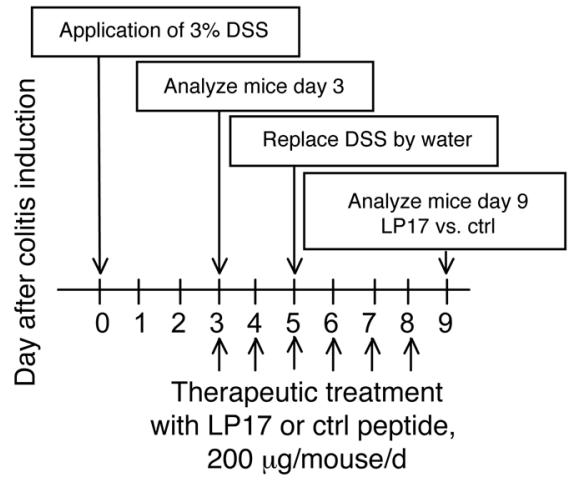

D

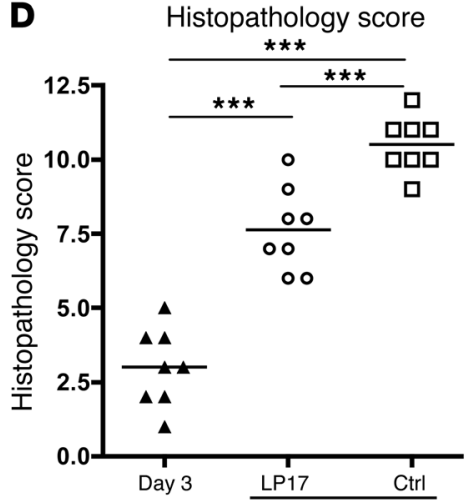

B

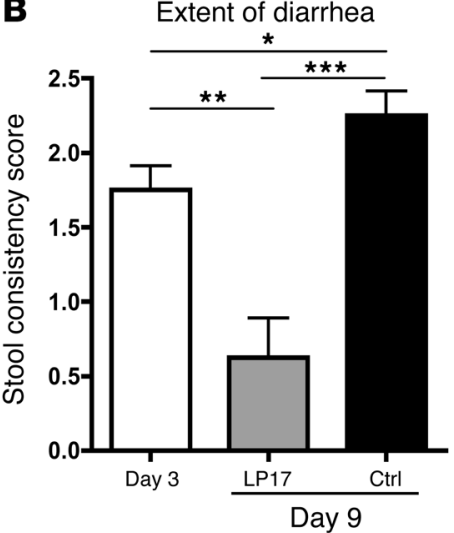

E Real-time RT-PCR analysis

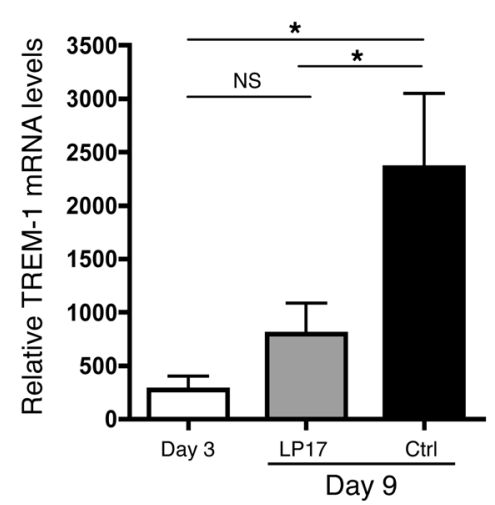

C Shortening of the colon
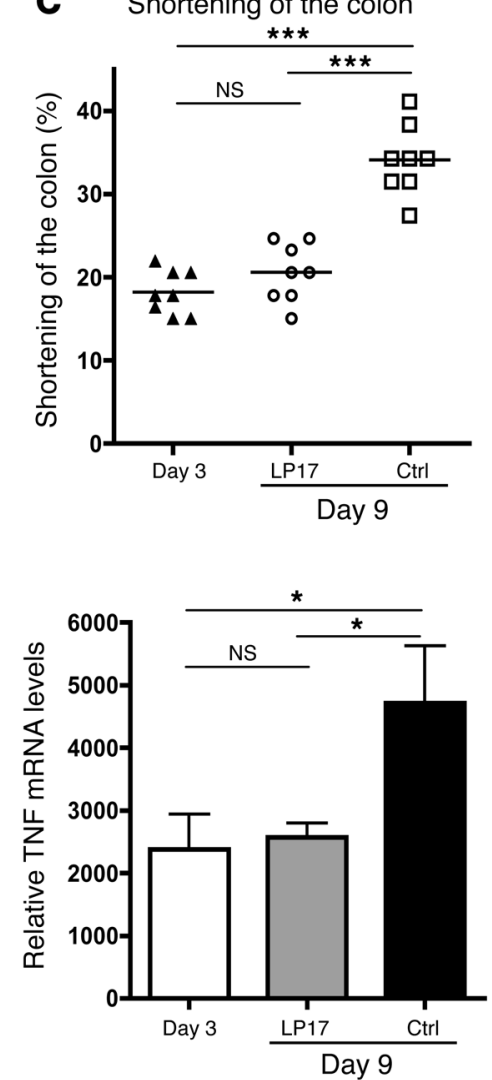

Figure 7

Blocking TREM-1 attenuates the disease process in established colitis. (A) When after oral administration of DSS the experimental mice developed colitis with persistent diarrhea and fecal samples had tested positive for the presence of occult blood (day 3 ), the first group of mice was analyzed (day $3 ; n=8$ ), and the remaining experimental mice were treated daily with either antagonistic TREM-1 peptide (LP17; $n=8$ ) or with control peptide $(n=8)$ for an additional 5 days. (B) Fecal samples from each mouse were tested for the presence of occult blood and bloody diarrhea to obtain the stool consistency score (see Methods). Data are indicated as mean $\pm \mathrm{SEM}$; $n=8$. (C) The colonic length in each mouse was measured from the end of the cecum to the anus; indicated are the mean loss of colon length (\%) on day 3 and on day 9 for both the LP17treated and control-treated groups. (D) Colitis scores for the 3 groups of mice were determined as described in Methods. (E) Real-time RT-PCR analyses for TREM-1 and TNF mRNA were performed individually on identical segments of the distal colon from each mouse in all experimental groups. ${ }^{\star \star \star} P<0.001 ;{ }^{*} P<0.01 ;{ }^{\star} P<0.05$.

treated with the antagonistic LP17 peptide showed only moderate signs of histopathological alterations, with a significantly lower colitis score (Figure 7D).

Tissue samples from identical distal parts of the colon from all mice were used to compare TREM- 1 and TNF mRNA expression by real-time RT-PCR. Control peptide-treated colitic mice showed significantly higher expression of both TREM-1 and TNF mRNA in the affected colon compared with LP17-treated mice. When the treatment with the antagonistic peptide LP17 was initiated at the time of overt colitis on day 3, expression of TREM-1 and TNF thereafter did not further increase and was significantly lower in LP17 treated mice than in controls at the end of the observation period (day 9) (Figure 7E). Therefore, modulation of TREM-1 engagement might represent a novel therapeutic approach for the treatment of IBD.

\section{Discussion}

IBD refers to 2 chronic diseases, CD and UC. Investigations in patients with IBD and experimental animal models of chronic intestinal inflammation suggest that IBD may occur in genetically pre- disposed individuals as a result of dysregulated immune response to constituents of the gut lumen, in particular to the intestinal flora that may occasionally penetrate the intestinal mucosa (8-10). Numerous reports contributed to the current concept that aberrant $\mathrm{T}$ cell functions including decreased regulatory activities or active functionally polarized $\mathrm{T}$ cells may contribute to the pathogenesis of IBD. Recent findings, including the demonstration of the NOD2/ CARD15 gene as a susceptibility locus for IBD (19), however, indicate that defects in the local innate immune response may predispose to onset of IBD. Quantitative and qualitative alterations in the pattern of secreted mediators produced by local macrophages may thus represent a major factor contributing to the pathogenesis of IBD.

Production and secretion of proinflammatory mediators in macrophages and neutrophils are also regulated via the TREM-1/ DAP12 signaling pathway. These effects of TREM-1 were previously demonstrated during acute inflammatory responses to microbial infections, e.g., in patients with bacterial pneumonia (20) and septic shock, and in mouse models of sepsis (2, 5, 21-23). Such proinflammatory effects of TREM-1 may be further enhanced in 
the presence of TLR-mediated signaling (24). In the seminal report on the involvement of TREM-1 in acute microbial inflammation, tissue specimens from patients with nonmicrobial, chronic inflammatory disorders such as psoriasis, UC, and immune complex-mediated vasculitis were analyzed by immunohistochemistry. However, in these specimens only rare TREM-1-positive cells were detected (2). Our previous analysis of TREM-1 expression in macrophages of the normal intestine revealed that TREM-1 is generally absent in the normal intestine (ref. 3 and Figures 1 and 2). This prompted us to reevaluate TREM-1 expression in the affected sites of patients with active IBD and to assess how disease activity relates to TREM-1 expression. Immunostaining (Figure 1), FACS analyses (Figure 2), and analysis of mRNA expression (Figure 4) clearly demonstrated a significant increase in TREM-1-expressing macrophages in the affected lesions of IBD patients. In the 2 different mouse models of colitis that we studied, and in patients with IBD, increased intestinal TREM-1 expression positively correlated with enhanced disease activity (Figures 4 and 5). Hence, the absence of large numbers of TREM-1-expressing macrophages in UC initially reported (2) may have been caused by low disease activity in the tissue samples analyzed. Furthermore, in the normal intestinal mucosa, TREM-1-expressing macrophages were largely absent. The frequency (and number) of TREM-1-expressing intestinal macrophages dramatically increased in IBD. However, even in the most active IBD lesions, the majority of the intestinal macrophages were still negative for TREM-1 expression (Figure 2).

In contrast to previous reports that focused on the importance of TREM-1 in septic shock and acute inflammation $(2,4-7)$, our results demonstrate a crucial role of TREM-1 during chronic inflammatory disorders of the intestine. Activation of TREM-1 on intestinal macrophages may have detrimental consequences for the perpetuation of intestinal inflammation and subsequent tissue destruction due to the several-fold increase in the level of proinflammatory mediators produced in vitro upon engagement of the TREM-1 (Figure 3). It has been possible to show significant protection of mice treated with the TREM-1 fusion protein (mTREM-1-hFc 1 ) against lethal E. coli-induced peritonitis and cecal puncture-induced shock, whereas treatment with TNFRI$\mathrm{hFc} \gamma 1$ even caused accelerated death of all animals (2). Thus, blocking TREM-1 substantially attenuates inflammatory responses but still allows sufficient control of bacterial infections. This may be relevant in the treatment of patients with chronic inflammatory disorders, since treatment of CD or rheumatoid arthritis with infliximab, a humanized antibody against TNF, is complicated by an increased risk for reactivation of a latent $M$. tuberculosis infection (25). Our results confirm that engagement of TREM-1 leads to a dramatic increase in secreted proinflammatory mediators known to contribute to the sustained inflammation and exacerbation of the disease. Blocking TREM-1 during experimental colitis attenuates the production of macrophage-derived crucial inflammatory mediators. This is also illustrated by the striking reduction in TNF mRNA levels in the colon of mice treated with an antagonistic TREM-1 peptide upon colitis induction (Figure 6). Since interfering with TREM-1 engagement led to the simultaneous reduction in production and secretion of a variety of proinflammatory mediators, TREM-1 may represent an attractive target for treating chronic inflammatory disorders.

Recently, increased serum levels of sTREM-1 were reported in patients with moderate or severe UC and CD (26). This further indicates that increased TREM-1 expression may closely reflect the extent of intestinal inflammation and may thus represent an elegant tool to monitor disease activity.

At present, TREM-1-deficient mice are not yet available to directly assess the consequences of the absence of TREM-1-mediated amplification of inflammation on the course of experimental colitis. Alternatively, the use of mice deficient for DAP12 $(27,28)$, i.e., the signal-transducing molecule associated with TREM-1, might be considered. However, DAP12 also associates with several other receptors, including TREM-2, which is coexpressed together with TREM-1 on some macrophage subsets. Therefore, results obtained with DAP12 $/-$ mice might be difficult to interpret with regard to the exact contribution of TREM-1-mediated effects to the inflammatory process.

Hence, we decided to use an antagonistic TREM-1-derived peptide to assess the contribution of TREM-1-mediated effects on the course of experimental colitis. Peptide treatment has the advantage of lower antigenicity compared with fusion proteins or antagonistic antibodies, especially in long-term treatment. A considerable disadvantage, however, is the short half-life of peptides in vivo. Hence, the moderate signs of inflammation still seen in experimental mice treated with the antagonistic TREM-1 peptide may be attributed to the inactivation of the antagonistic peptide in vivo.

The importance of an appropriate functional differentiation of intestinal macrophages is also illustrated by the spontaneous onset of colitis in several mutant mouse strains in which the differentiation of the macrophages is affected, e.g., through targeting IL-10 production in macrophages $(29,30)$. The origin of the TREM-1-expressing macrophages present in increased frequencies and numbers in the active lesions of patients with IBD and in mice with experimental colitis is still unclear (31-33). The coexpression of CD14 and CD89 on TREM-1-positive intestinal macrophages suggests that this inflammatory macrophage subset may represent exudate macrophages recently recruited to the site of inflammation. This circumstantial evidence is also supported by our findings in a mouse model of colitis: When colitogenic $\mathrm{CD}^{+} \mathrm{T}$ cells were transferred into $\mathrm{RAG} 2^{-/-}$recipients heterozygous for the gene encoding the fractalkine receptor $\left(\mathrm{CX}_{3} \mathrm{CR} 1\right)$ (1 allele was replaced with the gene encoding GFP; RAG2-/-CX ${ }_{3} \mathrm{CR} 1^{\mathrm{GFP} /+}$ mice) (34), the number of circulating monocytes increased and the ratio of $\mathrm{CX}_{3} \mathrm{CR} 1^{\text {lo }}$ to $\mathrm{CX}_{3} \mathrm{CR} 1^{\text {hi }}$ monocytes, representing inflammatory and resident monocytes, respectively (35), shifted from a 1:1 ratio in healthy animals to a $2: 1$ to $3: 1$ ratio in colitic mice. Similarly, the ratio of $\mathrm{CX}_{3} \mathrm{CR} 1^{\text {lo }}$ to $\mathrm{CX}_{3} \mathrm{CR} 1^{\text {hi }}$ macrophages and the total number of macrophages in the colonic lamina propria dramatically increased during onset of colitis. In colitic RAG2 ${ }^{-/-} \mathrm{CX}_{3} \mathrm{CR} 1^{\mathrm{GFP} /+}$ mice, TREM-1-expressing macrophages in the affected colonic mucosa were predominantly of the $\mathrm{CX}_{3} \mathrm{CR} 1^{\text {lo }}$ phenotype (data not shown).

Unfortunately, the transfer of CD 45.2 congenic monocyte subsets $\left(\mathrm{Gr}^{+}{ }^{+}\right.$versus $\left.\mathrm{Gr} 1^{-}\right)$into CD45.1 RAG2-/- recipients together with $\mathrm{CD}^{+} \mathrm{T}$ cell-mediated induction of colitis to directly determine the origin (i.e., resident macrophages versus recruited monocytes) of TREM-1-expressing colonic macrophages did not yield conclusive results, since only small numbers of macrophages of donor origin were recovered from the site of active colonic inflammation (data not shown). In addition, in the presence of a functional myelopoiesis in the recipient, transferred monocytes are rapidly outnumbered by host-derived monocytes, which, upon recruitment at the site of colonic inflammation, cannot be distinguished from the local, host-derived, resident 
macrophage population. Such a low recovery of transferred monocytes from the colonic mucosa is consistent with the reported low recovery of adoptively transferred monocytes from other organs such as lung, spleen, liver, and brain after more than 4 days after adoptive transfer (35).

Obviously, future work is needed not only to identify the mechanisms that lead to increased frequencies of TREM-1-expressing intestinal macrophages, but also to define the internal ligands that would activate the receptor during chronic colitis. At this point, it is not known whether upregulation of TREM-1 is the result of positive signals acting on resident intestinal macrophages or the absence of repressor mechanisms acting during physiological conditions on resident macrophages and recruited monocytes. Furthermore, it remains an open question whether the inflammation-amplifying effects of TREM-1 are mediated through the enhanced chemotactic recruitment of inflammatory cells and/or the TREM-1-mediated, enhanced inflammatory activities of TREM-1-expressing myeloid cells.

In conclusion, TREM-1-expressing macrophages are significantly increased in chronic inflammatory reactions of the intestinal mucosa in both IBD entities, UC and CD. TREM-1 expression on intestinal macrophages in IBD amplifies and prolongs inflammation via the enhanced secretion of disease-promoting mediators. Blocking TREM-1 upon induction of experimental colitis attenuates intestinal inflammation even when the antagonistic peptide is administered only after the first appearance of clinical signs of colitis. Hence, TREM-1 expression in the intestinal mucosa may not only be a reliable marker to assess disease activity in patients with IBD, but may also represent a promising target for future therapies for excessive acute inflammatory reactions as well as for chronic inflammatory diseases.

\section{Methods}

Human tissues. Normal small and large intestinal tissue specimens were obtained from patients who had undergone either colonoscopy with negative polyp screen and were otherwise healthy or abdominal surgery for cancer; or had localized infectious diseases. The tissue samples were taken at the furthest distance from the macroscopically apparent pathology, close to the resectional line in a healthy bowel area. The absence of histopathological signs of inflammation or neoplasia was confirmed by the assessment of H\&E-stained sections from paraffin-embedded tissue samples adjacent to the tissue specimen used for cell isolation. Actively inflamed intestinal tissue samples and biopsies were obtained from both UC and CD patients undergoing surgery or endoscopy. For cell isolation, tissue samples from the macroscopically affected part of inflamed intestinal lesions were used, and histopathological signs of active disease were confirmed by H\&E-stained sections of paraffin-embedded tissue samples adjacent to the specimens used for cell isolation. Additional biopsies were taken from uninvolved areas with a macroscopically normal appearance from UC and CD patients away from the diseased area as comparison. Tissue samples and biopsies were obtained according to institutional guidelines of the University Hospital Bern with prior informed consent of the patients. These procedures were also approved by the ethical committee of the state of Bern.

Cell isolation. Intestinal macrophages were isolated from human intestinal tissue samples and biopsies as previously described $(3,36)$. Briefly, small pieces of intestinal mucosa were incubated with $5 \mathrm{mM}$ EDTA/2 mM DTT, followed by digestion with $200 \mathrm{U} / \mathrm{ml}$ collagenase (Type IV; Sigma-Aldrich) plus $50 \mathrm{U} / \mathrm{ml}$ DNAse (Type I, grade II; Roche). Macrophages were enriched in a Percoll density gradient and subsequently purified using a depleting MACS procedure. As a control, monocytes were subjected to the same experimental conditions as intestinal macrophages during the entire isolation and fractionation procedure. These isolation steps, however, did not substantially influence cell-surface expression of CD14, CD33, HLA-DR, CD45, CD89, and TREM-1 (data not shown).

Flow cytometry. Fluorochrome-conjugated mAb against CD14 (TUK4; Serotec), CD33 (WM53; BD Biosciences - Pharmingen), HLA-DR (LN3; eBioscience), CD89 (A59; BD Biosciences - Pharmingen), and CD45 (HI30; BD Biosciences - Pharmingen) were used for FACS stainings. Supernatant of the human TREM-1-specific hybridoma 21C7 (4) and, as a second step, FITC-conjugated goat anti-mouse IgG1 (Sigma-Aldrich) were used for flow cytometric detection of TREM-1. Isotype controls with irrelevant specificities were included as negative controls. Octagam (1:2 in PBS; Novartis) was used to block Fc receptors and unspecific binding. At least $1 \times 10^{5}$ events were acquired on a FACScan LSRII (BD Biosciences) for subsequent analysis using FlowJo software (Tree Star). For analysis, scatter gates were first set for monocytes/macrophages upon backgating on $\mathrm{CD} 33^{+}$cells.

Immunohistochemistry. Serial frozen tissue sections $(5 \mu \mathrm{m})$ were fixed with acetone and rehydrated in TBS. Fc receptors and unspecific binding were blocked with $1 \%$ Octagam (Novartis) in TBS/BSA. As primary mAbs, anti-CD68 (EMB11; Dako) and supernatant of mouse anti-human TREM-1 hybridomas 17A11 and 42A7 (2) were used. As secondary antibody, a biotinylated rabbit anti-mouse IgG (E 354; Dako) was used, followed by an incubation step with the ABComplex-AP (Dako). Substrate solution (Naphthol AS-Bi phosphate, new fuchsin) was prepared according to the manufacturer's recommendations. Counterstaining was performed for 1 minute in hematoxylin.

Immunofluorescence stainings. Frozen tissue sections were fixed, rehydrated, and blocked as described for immunohistochemical stainings. As primary mAbs, anti-CD68-R-PE (clone Y1/82A; BD Biosciences - Pharmingen) and supernatant of the mouse anti-human TREM-1 hybridoma (17A11, 42A7; ref. 2) were used. As secondary reagent for the detection of anti-TREM-1, an anti-mouse IgG1-Alexa Fluor 488 conjugate (Molecular Probes; Invitrogen) was used.

Cell culture and subsequent cytokine measurement. Isolated intestinal macrophages were stimulated for 24 hours in 96-well flat-bottom plates coated with either anti-human TREM-1 (clone 21C7, mouse IgG1; ref. 4) or an isotype control (mouse IgG1; BD Biosciences - Pharmingen). Cells were plated at a concentration of $10^{6} \mathrm{cells} / \mathrm{ml}$, and subsequently, supernatants were collected and simultaneously tested for secreted TNF, IL-6, IL-8, MCP-1, and IL-1 $\beta$ using the luminex assay system (human x-Plex assays; Bio-Rad). Survival of the cells at the end of the in vitro culture was greater than $90 \%$ as assessed by trypan blue staining of the cultured cells.

Mice. C57BL/6 mice, purchased from Harlan, and C57BL/6 RAG2 $\%$ mice, bred in our specific pathogen-free (SPF) animal facility, were used at 8-12 weeks of age. All experimental mice were kept in microisolater cages in laminar flows under SPF conditions in the central animal facility of the Medical School, University of Bern. All animal experiments were reviewed and approved by the Animal Experimentation Review Board of the State of Bern.

Mouse models of colitis. For experiments involving the adoptive $\mathrm{T}$ cell transfer model, colitis was induced in C57BL/6 RAG2 /- mice by adoptive transfer of sorted $\mathrm{CD} 4{ }^{+} \mathrm{CD} 45 \mathrm{RB}^{\text {high }} \mathrm{T}$ cells, as previously described (12-14). Briefly, CD4 ${ }^{+}$ $\mathrm{T}$ cells were isolated from splenocytes from C57BL/ 6 mice, and after osmotic lysis of erythrocytes, $\mathrm{CD} 4^{+} \mathrm{T}$ cells were enriched by a negative MACS procedure for $\mathrm{CD} 8 \alpha$ and B220 (purified, biotinylated, hybridoma supernatant) using avidin-labeled magnetic beads (Miltenyi Biotec). Subsequently, the $\mathrm{CD} 4^{+} \mathrm{T}$ cell-enriched fraction was stained and FACS sorted for CD4+ (RM4-5; BD Biosciences - Pharmingen), CD45RB hi (16A; BD Biosciences - Pharmingen), and CD25- (PC61; eBioscience) naive T cells. Each C57BL/6 RAG2-/- mouse was injected i.p. with $1 \times 10^{5}$ syngeneic $\mathrm{CD} 4^{+} \mathrm{CD} 45 \mathrm{RB}^{\text {high }} \mathrm{CD} 25^{-} \mathrm{T}$ cells. Colitic mice were sacrificed and analyzed on day 14 after adoptive transfer. 
For experiments involving the DSS colitis model, C57BL/6 mice were given autoclaved tap water containing $3 \%$ DSS (DSS salt, reagent grade, mol wt: $36-50 \mathrm{kDa}$; MP Biomedicals) ad libitum over a 5-day period. The consumption of $3 \%$ DSS was measured. DSS was replaced thereafter by normal drinking water for another 4 days. Mice were euthanized and analyzed at the end of the 9-day experimental period.

TREM-1 peptide treatment. Upon colitis induction, either starting on day 0 or after onset of colitis on day 3 (as indicated), mice were treated with either an antagonistic TREM-1 peptide, LP17 (LQVTDSGLYRCVIYHPP), or a sequence-scrambled control peptide (TDSRCVIGLYHPPLQVY), as previously described for septic shock models by Gibot and coworkers (5). The peptides were chemically synthesized as $\mathrm{COOH}$ terminally amidated peptides (Pepscan Systems). Mice were treated once daily with $200 \mu \mathrm{g}$ peptide, injected i.p. in $200 \mu \mathrm{l}$ saline.

Colitis scoring. At the end of the experiments, the colon length was measured from the end of the cecum to the anus. Fecal samples were tested for occult blood using hemo FEC (Roche) tests (score 0 , negative test; 1 , positive test and no rectal bleeding; 2 , positive test together with visible rectal bleeding). The colon was divided into 2 parts. From each mouse, identical segments from the distal and proximal colon were taken for protein and RNA isolation and histology, and frozen tissue blocks were prepared for subsequent analysis. Histological scoring of paraffin-embedded H\&Estained colonic sections was performed in a blinded fashion independently by 2 pathologists. To assess the histopathological alterations in the distal colon, a scoring system was established using the following parameters: (a) mucin depletion/loss of goblet cells (score from 0 to 3); (b) crypt abscesses (score from 0 to 3); (c) epithelial erosion (score from 0 to 1); (d) hyperemia (score from 0 to 2); (e) cellular infiltration (score from 0 to 3 ); and (f) thickness of colonic mucosa (score from 1 to 3 ). These individual histology scores were added to obtain the final histopathology score for each colon ( 0 , no alterations; 15 , most severe signs of colitis).

RNA isolation and RT-PCR. RNA was isolated from intestinal tissue samples preserved in RNAlater (QIAGEN), using the RNAeasy Mini Kit (QIAGEN). RT-PCR was performed with 400 ng RNA each, using the TaqMan Gold RT-PCR Kit (Applied Biosystems). Primers were designed as follows: mouse TREM-1, forward 5'-GAGCTTGAAGGATGAGGAAGGC-3' and reverse 5'-CAGAGTCTGTCACTTGAAGGTCAGTC-3'; mouse TNF, forward 5'-GTAGCCCACGTCGTAGCAAA-3' and reverse 5'-ACGGCAGAGAGGAGGTTGAC- $3^{\prime}$; mouse $\beta$-actin, forward $5^{\prime}$-TGGAATCCTGTGGCATCCATGAAAC- $3^{\prime}$ and reverse $5^{\prime}$-TAAAACGCAGCTCAGTAACAGTCCG-3'; human TREM-1, forward 5'-CTTGGTGGTGACCAAGGGTTTTTC-3' and reverse 5'-ACACCGGAACCCTGATGATATCTGTC-3'; human TNF, forward $5^{\prime}$-GCCCATGTTGTAGCAAACCC-3' and reverse $5^{\prime}$-TAGTCGGGCCGATTGATCTC-3'; human GAPDH, forward 5' - TTCACCACCATGGAGAAGGC-3' and reverse 5'-GGCATGGACTGTGGTCATGA-3'. PCR products were semiquantitatively analyzed on agarose gels.

Human TREM-1 and mouse TREM-1 and TNF expression was also assessed by real-time PCR using the TREM-1 QuantiTect primer assay system and QuantiTect SYBR green PCR Kit (both from QIAGEN). GAPDH was used to normalize TREM-1 and TNF expression levels. DNA was amplified on a 7500 Real-Time PCR system (Applied Biosystems), and the increase in gene expression was calculated using Sequence Detection System software (Applied Biosystems).

Western blot analysis. Protein samples were separated on a denaturing $12 \%$ acrylamide gel, followed by transfer to nitrocellulose filter and probing with the primary antibody. Anti-TREM-1 (polyclonal goat IgG, $0.1 \mu \mathrm{g} / \mathrm{ml}$; R\&D Systems) or anti-tubulin (clone B-5-1-2, 1:5,000; Sigma-Aldrich) was used as primary reagent. As secondary antibodies, HRP-labeled donkey anti-goat Ig (1:2,000; The Binding Site) and goat anti-mouse Ig (1:4,000; Sigma-Aldrich) were used. Binding was detected by chemiluminescence using a Super Signal West Pico Kit (Pierce).

Statistics. The unpaired 2-tailed Student $t$ test was used to compare groups; $P$ values less than 0.05 were considered significant.

\section{Acknowledgments}

We thank Marco Colonna for helpful discussions and providing crucial reagents, including TREM-1-specific mAbs; Nikolina Sekulic for her expert histopathological assessment of mouse colonic tissue sections; and Frédéric Geissmann for providing breeder pairs of the $\mathrm{CX}_{3} \mathrm{CR} 1-\mathrm{GFP}$ mice. We are indebted to Andrew Macpherson for valuable comments on the manuscript. This study was supported by grants from the Swiss National Science Foundation, the Horten Foundation (to C. Mueller), and the Swiss IBD cohort study (SIBDCS).

Received for publication October 10, 2006, and accepted in revised form June 26, 2007.

Address correspondence to: Christoph Mueller, University of Bern, Institute of Pathology, PO Box 62, Murtenstrasse 31, CH-3010 Bern, Switzerland. Phone: 41-31-632-8904; Fax: 41-31-381-8764; E-mail: christoph.mueller@pathology.unibe.ch.
1. Bouchon, A., Cella, M., Grierson, H.L., Cohen, J.I., and Colonna, M. 2001. Activation of NK cell-mediated cytotoxicity by a SAP-independent receptor of the CD2 family. J. Immunol. 167:5517-5521.

2. Bouchon, A., Facchetti, F., Weigand, M.A., and Colonna, M. 2001. TREM-1 amplifies inflammation and is a crucial mediator of septic shock. Nature. 410:1103-1107.

3. Schenk, M., Bouchon, A., Birrer, S., Colonna, M., and Mueller, C. 2005. Macrophages expressing triggering receptor expressed on myeloid cells-1 are underrepresented in the human intestine. J. Immunol. 174:517-524.

4. Bouchon, A., Dietrich, J., and Colonna, M. 2000. Cutting edge: inflammatory responses can be triggered by TREM-1, a novel receptor expressed on neutrophils and monocytes. J. Immunol. 164:4991-4995.

5. Gibot, S., et al. 2004. A soluble form of the triggering receptor expressed on myeloid cells- 1 modulates the inflammatory response in murine sepsis. J. Exp. Med. 200:1419-1426.

6. Gibot, S., et al. 2005. Surface and soluble triggering receptor expressed on myeloid cells-1: expres- sion patterns in murine sepsis. Crit. Care Med. 33:1787-1793.

7. Gibot, S. 2005. Clinical review: role of triggering receptor expressed on myeloid cells-1 during sepsis. Crit. Care. 9:485-489.

8. Hanauer, S.B., et al. 2002. Maintenance infliximab for Crohn's disease: the ACCENT I randomised trial. Lancet. 359:1541-1549.

9. Bouma, G., and Strober, W. 2003. The immunological and genetic basis of inflammatory bowel disease. Nat. Rev. Immunol. 3:521.

10. Macdonald, T.T., and Monteleone, G. 2005. Immunity, inflammation, and allergy in the gut. Science. 307:1920-1925.

11. Smith, P.D., et al. 2001. Intestinal macrophages lack CD14 and CD89 and consequently are downregulated for LPS- and IgA-mediated activities. J. Immunol. 167:2651-2656.

12. Powrie, F., Leach, M.W., Mauze, S., Caddle, L.B., and Coffman, R.L. 1993. Phenotypically distinct subsets of CD4+ $\mathrm{T}$ cells induce or protect from chronic intestinal inflammation in C. B-17 scid mice. Int. Immunol. 5:1461-1471.
13. Morrissey, P.J., Charrier, K., Braddy, S., Liggitt, D., and Watson, J.D. 1993. CD4+ T cells that express high levels of CD45RB induce wasting disease when transferred into congenic severe combined immunodeficient mice. Disease development is prevented by cotransfer of purified CD4+ T cells. J. Exp. Med. 178:237-244.

14. Corazza, N., Eichenberger, S., Eugster, H.P., and Mueller, C. 1999. Nonlymphocyte-derived tumor necrosis factor is required for induction of colitis in recombination activating gene (RAG) $2(-/-)$ mice upon transfer of CD4(+)CD45RB(hi) T cells. J. Exp. Med. 190:1479-1492.

15. Dieleman, L.A., et al. 1994. Dextran sulfate sodiuminduced colitis occurs in severe combined immunodeficient mice. Gastroenterology. 107:1643-1652.

16. Kitajima, S., Takuma, S., and Morimoto, M. 1999. Changes in colonic mucosal permeability in mouse colitis induced with dextran sulfate sodium. Exp. Anim. 48:137-143.

17. Ni, J., Chen, S.F., and Hollander, D. 1996. Immunological abnormality in $\mathrm{C} 3 \mathrm{H} / \mathrm{HeJ}$ mice with heritable inflammatory bowel disease. Cell Immunol. 
169:7-15.

18. Okayasu, I., et al. 1990. A novel method in the induction of reliable experimental acute and chronic ulcerative colitis in mice. Gastroenterology. 98:694-702.

19. Maeda, S., et al. 2005. Nod2 mutation in Crohn's disease potentiates NF-kappaB activity and IL1beta processing. Science. 307:734-738.

20. Gibot, S. 2006. Soluble triggering receptor expressed on myeloid cells and the diagnosis of pneumonia and severe sepsis. Semin. Respir. Crit. Care Med. 27:29-33.

21. Gibot, S., et al. 2005. Surface triggering receptor expressed on myeloid cells 1 expression patterns in septic shock. Intensive Care Med. 31:594-597.

22. Gibot, S., et al. 2004. Plasma level of a triggering receptor expressed on myeloid cells-1: its diagnostic accuracy in patients with suspected sepsis. Ann. Intern. Med. 141:9-15.

23. Knapp, S., et al. 2004. Cutting edge: expression patterns of surface and soluble triggering receptor expressed on myeloid cells- 1 in human endotoxemia. J. Immunol. 173:7131-7134.

24. Bleharski, J.R., et al. 2003. A role for triggering receptor expressed on myeloid cells-1 in host defense during the early-induced and adaptive phases of the immune response. J. Immunol. 170:3812-3818.

25. Keane, J., et al. 2001. Tuberculosis associated with infliximab, a tumor necrosis factor alpha-neutralizing agent. N. Engl. J. Med. 345:1098-1104.

26. Tzivras, M., et al. 2006. Role of soluble triggering receptor expressed on myeloid cells in inflammatory bowel disease. World J. Gastroenterol. 12:3416-3419.

27. Hamerman, J.A., et al. 2006. Cutting edge: inhibition of TLR and FCR responses in macrophages by triggering receptor expressed on myeloid cells (TREM)-2 and DAP12. J. Immunol. 177:2051-2055.

28. Turnbull, I.R., et al. 2006. Cutting edge: TREM-2 attenuates macrophage activation. J. Immunol. 177:3520-3524.

29. Takeda, K., et al. 1999. Enhanced Th1 activity and development of chronic enterocolitis in mice devoid of Stat 3 in macrophages and neutrophils. Immunity. 10:39-49.

30. Kamada, N., et al. 2005. Abnormally differentiated subsets of intestinal macrophage play a key role in Th1-dominant chronic colitis through excess pro- duction of IL-12 and IL-23 in response to bacteria. J. Immunol. 175:6900-6908.

31. Rogler, G., et al. 1997. Alterations of the phenotype of colonic macrophages in inflammatory bowel disease. Eur. J. Gastroenterol. Hepatol. 9:893-899.

32. Smith, P.D., Ochsenbauer-Jambor, C., and Smythies, L.E. 2005. Intestinal macrophages: unique effector cells of the innate immune system. Immunol. Rev. 206:149-159.

33. Smythies, L.E., et al. 2005. Human intestinal macrophages display profound inflammatory anergy despite avid phagocytic and bacteriocidal activity. J. Clin. Invest. 115:66-75. doi:10.1172/JCI200519229.

34. Jung, S., et al. 2000. Analysis of fractalkine receptor CX(3)CR1 function by targeted deletion and green fluorescent protein reporter gene insertion. Mol. Cell. Biol. 20:4106-4114.

35. Geissmann, F., Jung, S., and Littman, D.R. 2003. Blood monocytes consist of two principal subsets with distinct migratory properties. Immunity. 19:71-82.

36. Rogler, G., et al. 1998. Isolation and phenotypic characterization of colonic macrophages. Clin. Exp. Immunol. 112:205-215. 